\title{
Understanding landmark types in human navigation from a database perspective
}

\author{
Hao Lyu ${ }^{\mathrm{a},}$ *, Wangshu Wang ${ }^{\mathrm{b}}$ \\ ${ }^{a}$ Chair of Cartography, TU Munich, Munich, Germany, hao.lyu@tum.de \\ ${ }^{b}$ Research Group Cartography, Department of Geodesy and Geoinformation, TU Wien, Vienna, Austria, \\ wangshu.wang@tuwien.ac.at \\ * Corresponding author
}

Keywords: location-based services (LBS), navigation, landmark, route communication

\begin{abstract}
:
Delivering location based information to support human navigation in unfamiliar environments has been a long standing research topic in Location-based Service (LBS). Enriching route and direction communication with landmark information not only increases the efficiency of navigation applications but also facilitates spatial knowledge learning among users (Lovelace et al. 1999, Wunderlich \& Gramann 2018). Landmarks are salient geographic entities anchored at fixed positions in an environment. In wayfinding and navigation, landmarks serve for multiple purposes as and organizing concept that help people build up structured spatial knowledge representation and as navigational tools to support navigational decision-making (Sorrows \& Hirtle 1999). In previous work, landmarks are often conceptualized as reference points. However, in Geodatabase landmarks are not always represented as point features. Moreover, nonpoint features are also important references in route instructions, especially for pedestrian navigation. The signs, though often considered as point elements not only provide something about "here", but also directional information (Gibson 2009). In this work, we attempt to provide a categorization of landmark for route communications from a database perspective. The proposed view is intended to be extended to a landmark model to generate various route communication, such as verbal instructions or symbolic visualizations.
\end{abstract}

Landmarks may serve differently in route instructions according to their affordances e.g., rivers can be walked along or gone across and parks can be gone across. Deriving a landmark model to support route communication needs a comprehensive understanding of landmarks. Route communication delivers route information in terms of distance and orientation with proper actions. Landmarks on the one hand provides possible action information according to their affordances, on the other hand act as references to express distances and orientations. Generating route information requires a system to be able to derive the geometric and topologic relationship between landmarks, the route, and the user's current position. We propose to model landmarks in a Geodatabase as abstract features that are linked to concrete geo-features e.g., buildings, rivers, signs, and parks, and thus their geometric shapes can be point, line, area, and direction other than only point. Considering their geographic locations, landmarks can be categorized as local or global (Klippel et al. 2009). The two types of landmarks are distinguished on whether it requires to be approached w.r.t. a specific route. Based on the visibility of a landmark, it can be classified as an in-view landmark or a distant landmark. The in-view landmark can be observed from the current view (i.e., decided by current position and orientation), while the distant landmark is invisible from current view. Though invisible, providing distant landmark still has positive effect on orientation and navigation ( $\mathrm{Li}$ et al. 2014). When examining the relationship between a landmark and a route, the landmark may fall into either on-route or off-route category. A route consists of a sequence of decision points and route segments connecting decision points. At decision points, people need to make decisions on maintaining current direction or turning to another direction. On-route landmarks help users to determine current location and reorientation, while off-route landmarks usually provide orienteering information (Sorrows \& Hirtle 1999). The categories are not mutually exclusive; they can be overlapped. For example, a landmark can be a local off-route landmark, while a distant one that is invisible from the current view.

The categorization of landmarks is based on the geometric and topologic concerns. To derive a landmark's geographic and topologic information about a specific route, information of user's position, route, and landmarks should be retrieved from database. The location of landmarks can be retrieved by directory queries. Their relative distances can be retrieved by spatial distance queries on their geometries. In 2D case, the topologic relationship can be described by DE9IM (Egenhofer \& Franzosa 1991). The queries on the topologic relationship are also implemented in geo-database systems. Given the following example in Figure 1, three geographic entities are mentioned in the route instruction as landmarks, a high tower, a grocery store, and a town park. The route is calculated by the route determination service in navigation system, while the salience score of the objects can be retrieved from precomputed results. We omitted the landmark identification and selection process here to keep focus. The high tower is far from the neighbouring area of the route but still visible, so it is a distant, off-route, global landmark, and used for orienteering in route instructions. The grocery store is a local, on-route landmark, which is used to give relative orientation between a user and itself at 
the decision point. The town park has a 2D polygon geometry in this case, which is an area-shaped landmark. It can be classified as a local, on-route landmark as it intersects with the neighbouring area of the routes. The instruction "walk along the edge of the town park" indicates that the town park is used for determining location and maintaining direction.

In this work we propose to understand landmarks from their geometric shapes and topologic relationships with routes and user's location. Understanding landmarks from a database perspective is helpful to integrate landmark information in route instructions. We plan to further extend this work for a landmark data model and implement automatic methods to support generating landmark information in route instructions.
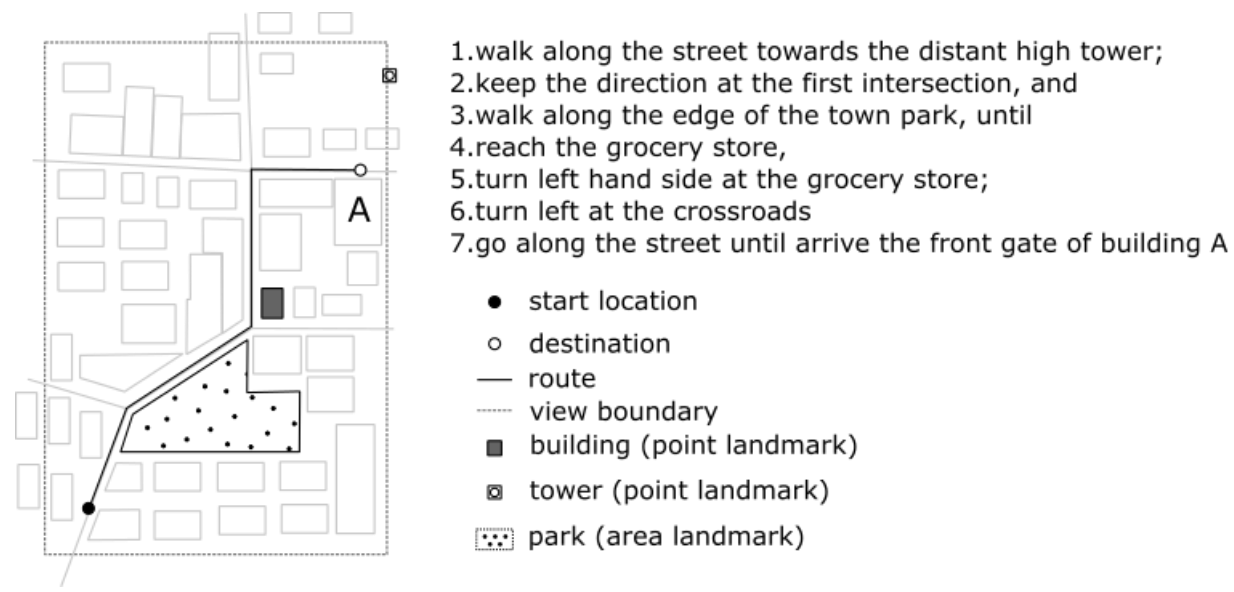

Figure 1. Visual (left) and verbal (right top) route instruction with landmark information in a wonder town.

\section{References}

Egenhofer, M. J., \& Franzosa, R. D. (1991). Point-set topological spatial relations. International Journal of Geographical Information System, 5(2), 161-174.

Gibson, D. (2009). The wayfinding handbook: Information design for public places. Princeton Architectural Press.

Klippel, A., Hansen, S., Richter, K. F., \& Winter, S. (2009). Urban granularities-a data structure for cognitively ergonomic route directions. GeoInformatica, 13(2), 223.

Li, R., Korda, A., Radtke, M., \& Schwering, A. (2014). Visualising distant off-screen landmarks on mobile devices to support spatial orientation. Journal of Location Based Services, 8(3), 166-178.

Lovelace, K. L., Hegarty, M., \& Montello, D. R. (1999, August). Elements of good route directions in familiar and unfamiliar environments. In International conference on spatial information theory (pp. 65-82). Springer, Berlin, Heidelberg.

Sorrows M.E., Hirtle S.C. (1999) The Nature of Landmarks for Real and Electronic Spaces. In: Freksa C., Mark D.M. (eds) Spatial Information Theory. Cognitive and Computational Foundations of Geographic Information Science. COSIT 1999. Lecture Notes in Computer Science, vol 1661. Springer, Berlin, Heidelberg

Wunderlich, A., Gramann, K. (2018 July) Navigation instructions including landmark information increase incidental spatial learning. In Conference: 3rd International Mobile Brain/Body Imaging Conference. 